\title{
A Study on Image Compression and its Applications
}

\author{
B. Reddaiah \\ Department of Computer Applications \\ Yogi Vemana University \\ Kadapa, A.P, India
}

\begin{abstract}
The fast development in digital filed gave scope for a lot of image processing applications. To make use of digital images efficiently, explicit methods are required to decrease the number of bits essential for their presentation. This guided for instant development in area of Image Processing. This increase forced to develop more strong algorithms. Compression concentrates on dropping image dimension without losing the originality and information present with original one. With this goal of compressing the image is to reduce redundancy present in image and to increase the storage capacity for well-organized communication. This research reviews important image compression methods. This paper discusses efficient techniques like lossy and lossless with its applications and research possibilities.
\end{abstract}

\section{Keywords}

Compression, Techniques, transmission, Lossy, Lossless, data, information,

\section{INTRODUCTION}

Image or data compression is some form of compression that is used to decrease the size of any kind of file. When the memory size of a file is reduced it requires less space to store and facilitates transmitting images in less time [2]. The fundamental form of compression process is shown in Fig 1.

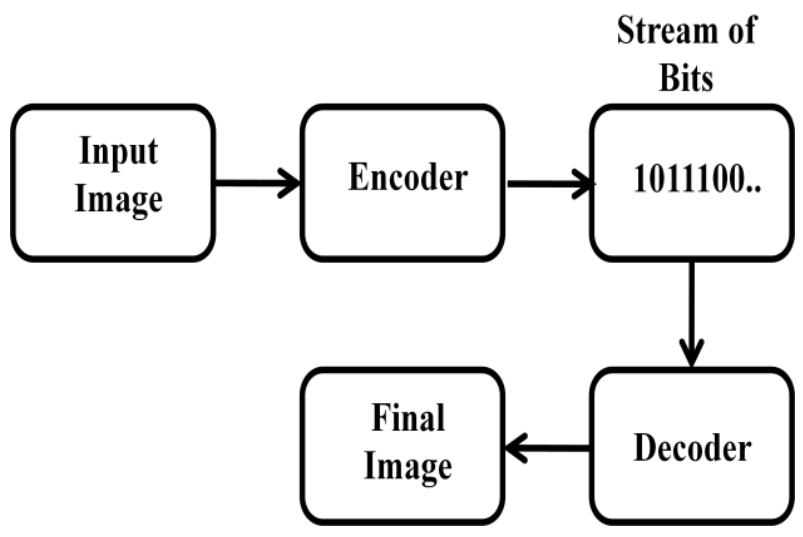

Fig: 1. Overview of Image Compression

Data Compression methods are basically of two types. The two methods are compression the data file and compression the media file.

\subsection{File Compression}

In this type of compression the size of a file or size of more number of files will be reduced. Once file compression is made on a file or files the consequential collection may reduce the disk space from 50 percent to 90 percent than that of original form. Different types of file compressions include
Zip, Gzip, RAR, and Stufflt and 7z. An exclusive algorithm for compression is used by each of the compression types for compressing the data.

\subsection{Media Compression}

As of file compression, the aim of compressing media file is to decrease the size of file so that the space in the disk can be saved. On the other hand, media compression algorithms are explicit to particular media kinds, like images, audio, and video files.

\section{NEED OF IMAGE COMPRESSION}

For single colored images there is no need of huge storage space to store the raw data. As the number of images to be stored increases or images to be transmitted increases the entire storage requirements become irresistible. Along with storage size, bandwidth for transmission and time taken for transmission of uncompressed images will be more and if compressed before its storage and transmission it may be proportional to the size of image. Before compressing the images as an initial step different types of redundancies are to be eliminated. In common, three basic digital images redundancies are in existence and the redundancies are as follows [3].

\subsection{Spatial Redundancy}

The values of neighboring pixels are strongly correlated in the majority of the natural images. From the values of neighboring pixels value of any given pixel can be predicted practically. This prediction by using any individual pixel is comparatively less significant and the redundancy can be reduced. The capability of the wavelets transformation is to efficiently capture variations at unusual scales. So, these wavelets are preferably suitable for this purpose.

\subsection{Coding Redundancy}

An image that is stored in the form of matrix, then the values range in between 0 to 255 and this frequency distribution of pixel values is called histogram. With this storage representation it is easy to find repeated times that each digit happens in the image. In standard images, some pixel values have larger frequency when compared to other pixels. In such cases coding redundancy is incurred when the same code word size is allocated to each pixel. Such redundancy can be reduced using Huffman coding. This is by assigning smaller number bits to the extra possible gray scale values than the less possible bits.

\subsection{Psycho visual Redundancy}

Psycho visual redundancy is based on the properties of human vision. Human eyes cannot respond to all visual information with equivalent sensitivity. Certain information is comparatively less importance when compared to additional information and typical visual processing is called psycho visual redundancy. 


\section{COMPRESSION TECHNIQUES}

Compression is a procedure where particular information is characterized with reduced number of data points. Image compression techniques as shown in Figure 2 and are broadly classified into two categories as lossy and lossless image compression $[5,4,1]$.

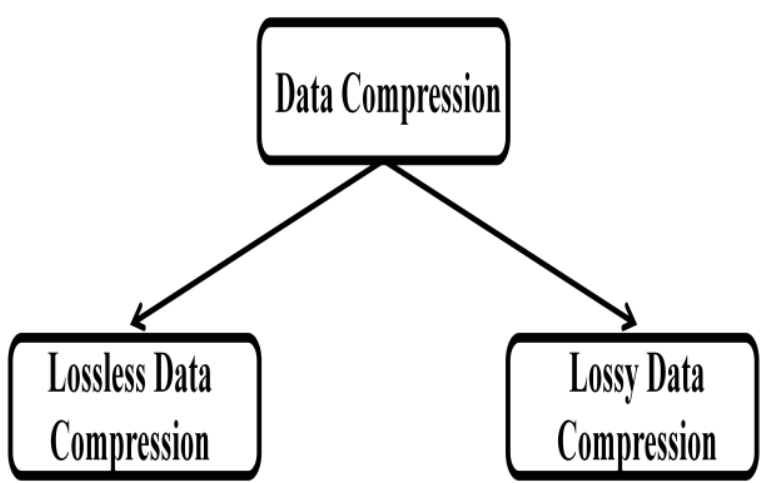

Fig: 2. Types of Data Compression Methods

These two different techniques have same aim and are used to compress files. The Lossy and lossless techniques has different methods that are used by different file formats for achieving results. Lossy compression methods are like Discreet Cosine Transform method, vector quantization method and Huffman coding method whereas Lossless compression methods like Run Length Encoding method and string table method. The compression is measured by the compression ratio $\left(\boldsymbol{C}_{\boldsymbol{R}}\right)$.

Mathematically the quantity that measures the quality of a reconstructed image is compared with the original image and is known as peak signal to noise ratio (PSNR), that is measured in decides $(\mathrm{dB})$.

\subsection{Lossless Compression}

This technique is generally applied for archival purposes. Images that have geometric shapes and are comparatively simple are considered for lossless compression. It is used for images that belongs to medical, technical drawings, clip art etc. In this compression technique the original data is absolutely reconstructed from the compressed data and used to size the file with no loss in image quality and is shown in the Figure 3.

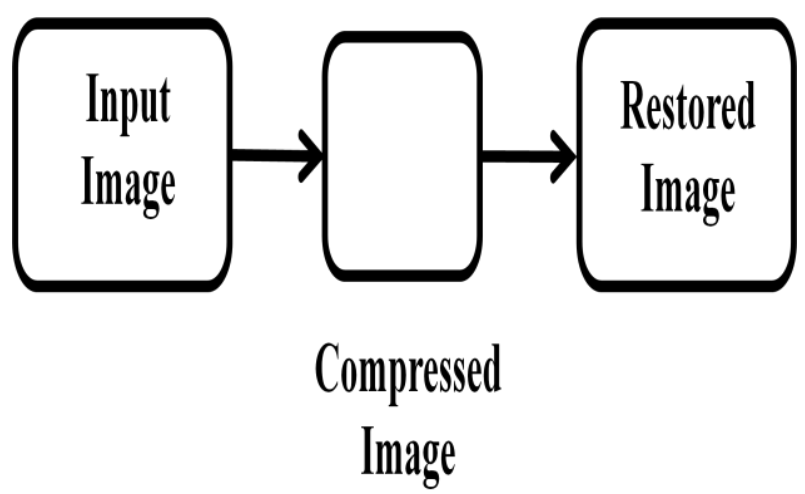

Fig: 3. No loss shown in Lossy Compression

While compressing all the information in the original images is used. This will help in decompression to get back to original form that is accurately equal to the input data and the process is shown in the Figure 4.

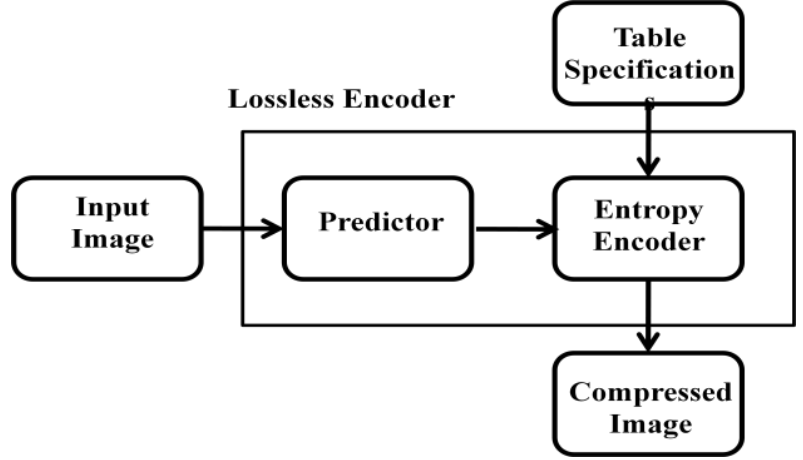

Fig: 4. Overview of Lossless Compression

Lossless compression can not only used for graphic images but it can be used for computer data files such as spreadsheets, text documents and other software applications like sending files as email attachments. While compressing text files for repeated letters a common format is used and for the short term they are discarded. Once the text file is decompressed, the letters are retrieved.

The major advantages of Lossless Compression techniques are that they are mainly useful for compressing large size files. Once the file is compressed when restoring the file back, exact data is restored after re-compression. After recompression high quality data is retrieved, so that there will be no difference between the original image and restored image.

The major disadvantages of Lossless Compression techniques are that once the image is compressed the ratio of compression will be low. After compression when the data is being transmitted the time taken to transfer the image is high. While reconstructing the image back after transmission decoding the image to get back the original image is a difficult process.

\subsubsection{Run Length Encoding}

Run length encoding is a lossless compression technique that replaces data by a pair of values namely value that indicates the repeated value and length that indicates the number of repetitions. This compression technique is a simple one in which data is in the form of runs. Runs are sequences where same data value takes place in several successive data elements. They are stored as a single data value and count, rather than as the original run.

\subsubsection{Predictive Coding}

This technique predicts the value of each pixel by using the value of its neighboring pixels. As a result, each pixel is encoded with a prediction error rather than its original value. These errors to a great extent are smaller when compared to original value, so that lesser bits are required to store bits. Along with predictive coding another alternative predictive coding is adaptive coding. This technique splits the image into blocks. The prediction coefficient is computed independently.

\subsubsection{Arithmetic Encoding}

This encoding technique represents a message as some finite intervals between 0 and 1 . Based on the probabilities of the message symbols the interval is divided into smaller intervals. This technique is the most capable method to code symbols according to the probability of their occurrence.

\subsubsection{Entropy Encoding}

This technique minimizes the size of dataset required to communicate a particular amount of information. Entropy 
coding method is used in Huffman and arithmetic coding techniques. When there are repeated characters and symbols first they are noted down. After noting instead of repeating at every pixel, the positions of these pixels are recorded. Further same symbols are assigned.

\subsubsection{LZW Coding}

LZ technique replaces repetitive substrings to previous occurrences of strings. The repeated data is substituted by position and length of existing substring

\subsubsection{Huffman Coding}

Huffman Coding technique makes use of variable length code. Here short code words are allocated to most familiar values or for symbols in the data. Longer code words are allocated to less repeated occurring values. This technique generates less redundancy cods when compared to other techniques. This coding is used for compressing text, images, and videos.

\subsection{Lossy Compression}

Lossy compression methods are particularly used to compress natural images like photographs, where negligible loss for reliability is adequate to accomplish a considerable reduction in bit rate. This is most frequently used to compress multimedia data and some data may be lost. This is not suited and used for text based documents and software as they need to keep the exact information without loss. This technique allows higher compression ratio than the lossless technique. In this compression the rebuilding of an image is only an approximation of the original data and original image is not retrieved as it is and very small amount of data loss may be seen as shown in Figure 5.

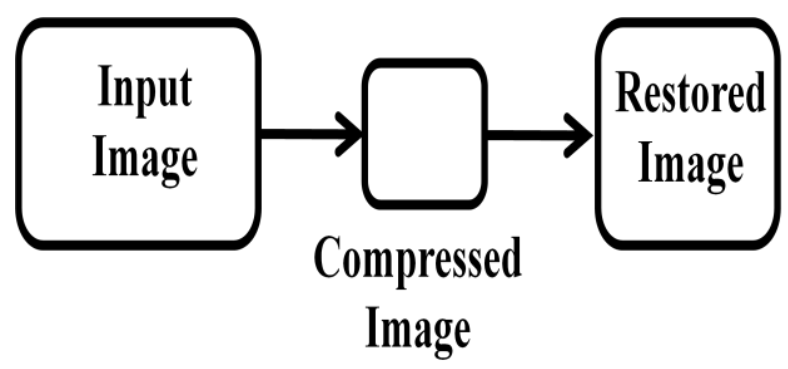

Fig: 5. Data loss shown in Lossy Compression

This type of compression also looks for redundant pixel information and that is permanently discarded. So when the file is retrieved back from compression the original data is not exactly retrieved. Even though when the exact data is not retrieved, data missing cannot be experienced. The process of lossy compression is shown in Figure 6 .

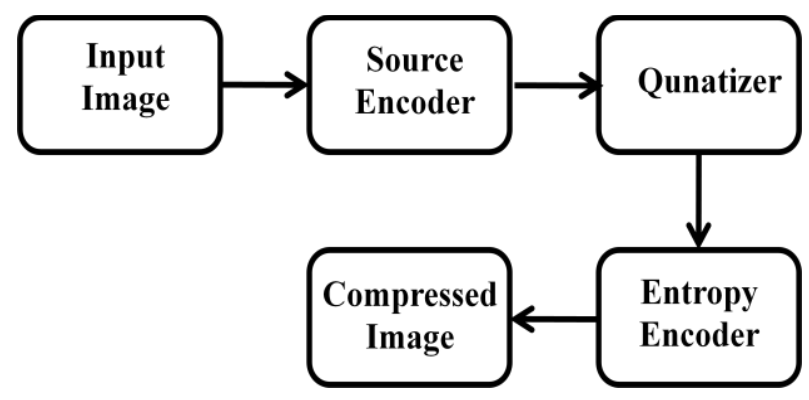

Fig:6 Overview of Lossy Compression

The major advantages of Lossy Compression techniques are that they are mainly useful for compressing small files. Once the files are compressed, they take very less time to send the files from one place to another and easy to move and store.
The files compressed by these methods are easily usable, quick to open and good for using in internet applications. The processed file are with low resolution, low quality and mostly free from threats.

The major disadvantages of Lossy Compression techniques are that they are not supportive for editing the images. In these images the editable data is dumped and have very minute archive value. The resolution of the processed images will be low by which the quality of printing the images may be limited.

\subsubsection{Discrete Cosine Transform}

Discrete Cosine Transform is a lossy Compression technique specifically used for compressing image and audio files. This technique translates data into the summation of series of cosine waves oscillating at different frequencies.

\subsubsection{Discrete Wavelet Transform}

Discrete wavelet transform characterizes image as a sum of wavelet functions. These functions are known as wavelets that have dissimilar location and scale. This technique is typically executed by means of hierarchical filter structure and it is applied on image blocks.

\subsubsection{Fractal Compression}

This technique depends on certain parts of images resembling other parts of same image. This algorithm converts these parts into mathematical data. This data is known as fractal codes that are used to reconstruct the encoded image. As the image is converted into fractal code it becomes resolution independent.

\subsubsection{Vector Quantization}

This technique is a fixed to fixed length algorithm called LBG-VQ algorithm. It divides a large set of data points called vectors into groups. It encodes values from a multidimensional vector space into a finite set of values to from a discrete subspace of lower dimension. This lower space vector needs less storage space and by which data is compressed. This technique is used for voice and images.

\section{APPLICATIONS OF COMPRSSION}

For image compression applications the above discussed Lossy Compression and Lossless compression methods are used. Various techniques and algorithms are used in compressing images by different image compression applications. Among these two methods, selection of a method to compress images depends on the quality of the output that is expected by the users. When a very high quality output is expected without any loss of data by users from image compression applications then lossless compression technique can be used by the application.

Lossless compression techniques are used when a high degree of accuracy is needed. In some cases where the quality is not that much important and can be compromised, lossy compression technique can be used. When this compression technique is used there will be very little bit of loss in quality and that loss cannot be visible to the human eye at most of times. It can be used in such applications where a little compromise on quality of image is tolerable. The areas where image compression is used are in television broadcasting, Remote sensing via satellite, for military communication systems through radars, Tele conferencing systems, communications systems built through computers, Facsimile transmission medical images in computer tomography magnetic resonance imaging, capturing and transmitting satellite images, geological surveys, weather reporting 
applications

\section{CONCLUSION}

In this study different image compression methods has been discussed. There is wide research going on in the field of compression. This is possible by keeping in view the requirement of low bit rate methods for compression this study has been carried out. This study has found that there is more scope for research in this filed to develop more efficient algorithms.

There is more demand for compression algorithms and it is found that the research is to be made keeping in mind peak signal to noise ratio and compression ratio for better algorithms

\section{REFERENCES}

[1] Richa Goyal, Jasmeen Jaura, "A Review of Various Image Compression Techniques", International Journal of Advanced Research in Computer Science and
Software Engineering, Volume 4, Issue 7, July 2014.

[2] Harpreet Kaur, Rupinder Kaur, Nvdeep Kumar, "Review of Various Techniques for Medical Image Compression", International Journal of Computer Applications, Volume 123, No. 4, Auguset 2015.

[3] Bhonde Nilesh, Shinde Sachin, Nagmode Pradip, D.B. Rane, "Image Compression Using Discrete Wavelet Transform”, IJCTEE, Volume 3, March-April 2013.

[4] A. Alarabeyyat, S. Al-Hashemi, T. Khdour, M. Hjouj Btoush, S. Bani-Ahmed, R. Al-Hashemi, "Lossless Image Compression Technique Using Combination Methods", Journal of Software Engineering and Applications, 2012.

[5] Malwinder Kaur, Navdeep Kaur, "A literature Survey on Losllless Image Compression”, Internatiional Journal of Advanced Research in Computer and Communication Engineering, Vol., 4, Issue , March 2015. 